\title{
High Contiguity Whole Genome Sequence and Gene Annotation Resource for Two Venturia nashicola Isolates
}

\author{
Maxim Prokchorchik, ${ }^{1}$ Kyungho Won, ${ }^{2}$ Yoonyoung Lee, ${ }^{1}$ Eu Ddeum Choi, ${ }^{2}$ Cécile Segonzac,,${ }^{3,4}$ \\ and Kee Hoon Sohn ${ }^{1,5, \dagger}$ \\ ${ }^{1}$ Department of Life Sciences, Pohang University of Science and Technology, Pohang 37673, Republic \\ of Korea \\ ${ }^{2}$ National Institute of Horticultural and Herbal Science (NIHHS), Rural Development Administration \\ (RDA), Naju, Republic of Korea \\ ${ }^{3}$ Department of Plant Science, Plant Genomics and Breeding Institute and Research Institute of \\ Agriculture and Life Sciences, College of Agriculture and Life Sciences, Seoul National University, \\ Seoul 08826, Republic of Korea \\ ${ }^{4}$ Plant Immunity Research Center, College of Agriculture and Life Sciences, Seoul National University \\ ${ }^{5}$ School of Interdisciplinary Bioscience and Bioengineering, Pohang University of Science and \\ Technology, Pohang 37673, Republic of Korea
}

\begin{abstract}
Venturia nashicola is a fungal pathogen that causes Asian pear scab disease. This pathogen is of particular importance in Northeast Asian countries, where Asian pears are grown industrially. Scab disease in Asian pear is currently controlled by fungicide spraying and this situation calls for developing scab resistant cultivars. High-quality genome data are therefore required for in-depth comparative genome analysis of different isolates of $V$. nashicola and $V$. pyrina, a closely related species, which only infects European pear plants. Here, we report the high-contiguity whole genome assembly of two $V$. nashicola isolates, which is expected to enable genome comparisons for identification of the genes involved in host range determination of $V$. nashicola.
\end{abstract}

\section{Genome Announcement}

Venturia nashicola Tanaka \& Yamamoto, a causal agent of scab disease in Asian pears (e.g., Pyrus pyrifolia, P. ussuriensis, P. betulaefoila, and P. bretschneidieri) (Abe et al. 2008; Ishii and Yanase 2000; Li et al. 2007; Tanaka and Yamamoto 1964), is a major threat for the pear industry in Northeast Asia. Although, V. nashicola is limited to countries where Asian pears are grown industrially, including Japan, Korea, Taiwan, and China (Plantwise database), it is considered as a critical quarantine threat in thye European Union, United States, Israel, and Turkey (Jeger et al. 2017). As there is no commercially available resistant cultivar, scab disease is controlled by multiple fungicide applications every year (Ishii and Yanase 2000; Park et al. 2000). Unfortunately, development of $V$. nashicola resistance to the commonly used fungicides has been reported (Ishii 2011).

The genus Venturia is phylogenetically assigned to the order Venturiales within the class Dothideomycetes (Zhang et al. 2011). Species of this genus can infect hosts in six plant

Maxim Prokchorchik and Kyungho Won contributed equally.

${ }^{\dagger}$ Corresponding author. K. H. Sohn; khsohn@postech.ac.kr

The author(s) declare no conflict of interest.

Accepted for publication 18 April 2019.
Funding

This work was carried out with the support of "Cooperative Research Program for Agriculture Science \& Technology Development" (Rural Development Administration project number PJ01191301) and a National Research Foundation of Korea grant funded by the Republic of Korea government (MSIT) (grant number 2018R1A5A1023599). C. Segonzac is supported by the Creative-Pioneering Researchers Program through Seoul National University.

\section{Keywords}

fungus-plant interactions, genomics 
Table 1. Statistics of the whole genome assemblies of Venturia nashicola isolates

\begin{tabular}{lcc} 
& \multicolumn{2}{c}{ Venturia nashicola } \\
\cline { 2 - 3 } Assembly parameters & MAFF615029 & PRI2 \\
Sequencing technology & Pac-Bio & Illumina \\
Sequencing coverage & $79 \times$ & $220 \times$ \\
Package used for assembly & $H G A P 2$ & bbmap \\
Number of a contigs or ${ }^{\mathrm{b}}$ scaffolds & $198^{\mathrm{a}}$ & $55^{\mathrm{b}}$ \\
Total residues & $41,878,255$ & $40,199,691$ \\
Mean length & $211,506.34^{\mathrm{a}}$ & $730,903.5^{\mathrm{b}}$ \\
Minimum length & $3,885^{\mathrm{a}}$ & $9,793^{\mathrm{b}}$ \\
Maximum length & $2,819,867^{\mathrm{a}}$ & $2,807,685^{\mathrm{b}}$ \\
L50 & $1,515,729^{\mathrm{a}}$ & $1,787,749^{\mathrm{b}}$ \\
L90 & $469,434^{\mathrm{a}}$ & $711,231^{\mathrm{b}}$ \\
NN content & 0 & 158,627 \\
GC content (\%) & 46.2 & 46 \\
Repeat content of genome (\%) & 2.5 & 2.5 \\
Number of predicted genes & 12,163 & 11,760 \\
Percentage completeness & 98.3 & 97 \\
\hline
\end{tabular}

a Statistic provided is that of a contig.

b Statistic provided is that of a scaffold.

families, including Acaraceae, Betulaceae, Cornaceae, Oleaceae, Rosaceae, and Salicaceae (Sivanesan 1977). In general, Venturia species are host-specific pathogens and each can only infect one plant genus or close relatives in a given host family (González-Domínguez et al. 2017). The best studied and most economically damaging species is $V$. inaequalis, which causes scab disease in apple (Bowen et al. 2011; MacHardy 1996). In contrast, fewer studies have been reported for related Venturia species such as $V$. nashicola, V. pyrina, and $V$. carpophila infecting Asian pear, European pear, and peach, respectively (Ishii and Yanase 2000; Sivanesan 1974; Sivanesan and Waller 1974). V. nashicola and V. pyrina were initially considered as one species but were later separated based on distinct morphological and pathological characteristics (Ishii and Yanase 2000). Moreover, V. nashicola and V. pyrina are unable to cause disease in European pear and Asian pear, respectively, indicating that the pear scab pathogens have highly restricted host ranges (Abe et al. 2008; Ishii and Yanase 2000; Park et al. 2000). Recently, it was shown that disease resistance $(R)$ genes play a major role in the nonhost resistance of Arabidopsis thaliana to Albugo candida (white rust) (Cevik et al. 2019). However, the extent to which effector-triggered $R$ gene-dependent immunity plays a role in nonhost resistance of European and Asian pear to $V$. nashicola and $V$. pyrina, respectively, is currently unknown. A draft genome of $V$. pyrina has been recently published (Deng et al. 2017). The draft genome sequence and annotation of $V$. nashicola isolate Yasato 2-1-1 was reported recently (Johnson et al. 2018). However, since this assembly seems to be highly fragmented (40,800 scaffolds), we aimed to provide improved Venturia nashicola genome assembly and annotations.

To prepare the DNA samples for genome sequencing, Venturia nashicola isolates MAFF615029 and PRI2, sampled from scab-infected pear in Japan and the Republic of Korea, respectively, were grown on sterile Miracloth filter paper placed on potato dextrose agar media at $20^{\circ} \mathrm{C}$ for 30 days before mycelia were harvested and ground with liquid nitrogen for genomic DNA extraction, using the CTAB method (Doyle 1987) followed by purification on NucleoSpin columns (Macherey-Nagel $\mathrm{GmbH} \& \mathrm{Co}$. KG). For $V$. nashicola isolate MAFF615029, $5 \mu \mathrm{g}$ of genomic DNA was used for construction of a single-molecule real-time (SMRT) bell library (Pacific Biosciences). Small fragments less than $20 \mathrm{~kb}$ of SMRT bell template were removed, using BluePippin size selection system for large-insert library. Subsequently, the SMRT bell library of MAFF615029 isolate was sequenced using the PacBio RS II sequencing platform. We aimed to use the PacBio sequenced MAFF615029 genome sequence as a reference for assembly of the PRI2 genome that was sequenced using the Illumina Hi-seq platform.

For $V$. nashicola MAFF615029, genome assembly of the PacBio reads was performed using the HGAP2 assembly pipeline with post-assembly Quiver polishing at DNA-link (Seoul, Korea), which resulted in a high-contiguity genome assembly consisting of 198 scaffolds totaling $41,878,255$ base pairs (Table 1 ). Prior to assembly of the $V$. nashicola PRI2 genome, Illumina short reads were trimmed with Trimmomatic v0.38 (Bolger et al. 2014) and were assessed for quality, using FastQC (Andrews 2014). Illumina short reads were initially de novo assembled with the 
MaSuRCA assembler (Zimin et al. 2013), generating 1,849 contigs totaling 33,716,288 base pairs. This was significantly shorter than the PacBio-based assembly of the $V$. nashicola isolate, which yielded more than $40 \mathrm{Mb}$. This was expected, as short reads do not allow proper repeat region assembly and, overall, produce shorter de novo contigs. Therefore, a reference guided assembly of the $V$. nashicola PRI2 Illumina short reads was performed using bbMap, with $V$. nashicola isolate MAFF615029 PacBio assembly as a reference (Bushnell 2014). Further scaffolding was performed with Ragout (Kolmogorov et al. 2014) and polishing was done with Pilon (Walker et al. 2014), reusing the Illumina data. $V$. nashicola PRI2 genome assembly resulted in 55 scaffolds totaling 40,227,028 base pairs which is comparable to the MAFF615029 isolate (Table 1). The fact that the PRI2 assembly yielded fewer scaffolds than the MAFF615029 assembly is due to less stringent contig linking at the scaffolding step, allowing gaps to be filled with $\mathrm{N}$ bases.

Gene models were predicted using the modified version of the FunGAP gene annotation pipeline (Min et al. 2017). All the FunGAP steps relying on transcriptome data were changed to work without it or were removed from the pipeline. FunGAP takes advantage of several gene prediction tools, such as BRAKER (Hoff et al. 2016), MAKER (Cantarel et al. 2008) and AUGUSTUS (Stanke et al. 2006). Originally BRAKER requires transcriptome data; thus, we altered its subcommand to self-train based on the translated transcripts from $V$. inaequalis ICMP13258 gene prediction aided by its own transcriptome data (Deng et al. 2017). We also performed AUGUSTUS training on the same gene set (Deng et al. 2017). There were 12,163 genes predicted in $V$. nashicola MAFF615029 isolate and 11,760 genes predicted in $V$. nashicola PRI2 isolate. These numbers are comparable to the gene content reported for other Venturia genomes of similar size (Deng et al. 2017).

Completeness of genome assemblies and gene predictions were assessed using BUSCO (Simão et al. 2015). Assessment of genome completeness with the Ascomycota BUSCO dataset (1,325 BUSCO groups total) showed $98.3 \%$ completeness with 1,292 complete, 19 fragmented, and four missing BUSCO orthologs in MAFF615029 and 97\% completeness with 1,275 complete, 17 fragmented, and 23 BUSCO orthologs in PRI2.

In contrast, assessment with the BUSCO Fungi dataset (290 BUSCO groups) showed 98.6\% completeness with 286 complete, two fragmented, and two missing BUSCO orthologs in MAFF615029 and 97.9\% completeness with 284 complete, one fragmented, and five missing BUSCO orthologs in PRI2 (Table 1).

Assemblies of fungal genomes are challenging due to their size in the tens of megabases and the presence of genomic repeats. Short read-only based assemblies suffer from high rates of fragmentation and unresolved genomic repeats (Thomma et al. 2016). The high contiguity genome sequences of two $V$. nashicola isolates presented in this resource will provide a platform for genomic comparisons, phylogenetic analyses, and host determinant mining in order to understand the basis of high host specificity in Venturia spp. It can be further used, in conjunction with optical mapping, to achieve chromosome level assemblies of V. nashicola completed reference genome in future studies (Faino et al. 2015).

Full genome assembly data and genome annotations are deposited in the NCBI database under BioProject accession number PRJNA527174.

\section{Author-Recommended Internet Resource}

Plantwise database: https://www.plantwise.org

\section{Literature Cited}

Abe, K., Saito, T., Terai, O., Sato, Y., and Kotobuki, K. 2008. Genotypic difference for the susceptibility of Japanese, Chinese and European pears to Venturia nashicola, the cause of scab on Asian pears. Plant Breed. 127: 407-412.

Andrews, S. 2014. FastQC: A quality control tool for high throughput sequence data. Published online. http://www.bioinformatics.babraham.ac.uk/projects/fastqc

Bolger, A. M., Lohse, M., and Usadel, B. 2014. Trimmomatic: A flexible trimmer for Illumina sequence data. Bioinformatics 30:2114-2120.

Bowen, J. K., Mesarich, C. H., Bus, V. G., Beresford, R. M., Plummer, K. M., and Templeton, M. D. 2011. Venturia inaequalis: The causal agent of apple scab. Mol. Plant Pathol. 12:105-122.
Bushnell, B. 2015. BBMap: A fast, accurate, splice-aware aligner. Published online. http://www.sourceforge.net/projects/bbmap

Cantarel, B. L., Korf, I., Robb, S. M., Parra, G., Ross, E., Moore, B., Holt, C., Sánchez Alvarado, A., and Yandell, M. 2008. MAKER: An easy-to-use annotation pipeline designed for emerging model organism genomes. Genome Res. 18:188-196.

Cevik, V., Boutrot, F., Apel, W., Robert-Seilaniantz, A., Furzer, O. J., Redkar, A., Castel, B., Kover, P. X., Prince, D. C., Holub, E. B., and Jones, J. D. G. 2019. Transgressive segregation reveals mechanisms of Arabidopsis immunity to Brassica-infecting races of white rust (Albugo candida). Proc. Natl. Acad. Sci. U.S.A. 116:2767-2773.

Deng, C. H., Plummer, K. M., Jones, D. A. B., Mesarich, C. H., Shiller, J., Taranto, A. P., Robinson, A. J., Kastner, P., Hall, N. E., Templeton, M. D., and Bowen, J. K. 
2017. Comparative analysis of the predicted secretomes of Rosaceae scab pathogens Venturia inaequalis and $V$. pirina reveals expanded effector families and putative determinants of host range. BMC Genomics 18:339.

Doyle, J. J., and Doyle, J. L. 1987. A rapid DNA isolation procedure for small quantities of fresh leaf tissue. Phytochem. Bull. 19:11-15.

Faino, L., Seidl, M. F., Datema, E., van den Berg, G. C., Janssen, A., Wittenberg, A. H., and Thomma, B. P. 2015. Single-molecule real-time sequencing combined with optical mapping yields completely finished fungal genome. MBio 6:e00936-15.

González-Domínguez, E., Armengol, J., and Rossi, V. 2017. Biology and epidemiology of Venturia species affecting fruit crops: A review. Front. Plant Sci. 8:1496.

Hoff, K. J., Lange, S., Lomsadze, A., Borodovsky, M., and Stanke, M. 2016. BRAKER1: Unsupervised RNA-seq-based genome annotation with GeneMarkET and AUGUSTUS. Bioinformatics 32:767-769.

Ishii, H. 2011. Resistance in Venturia nashicola to benzimidazoles and sterol demethylation inhibitors. Pages 21-31 in: Fungicide Resistance in Crop Protection. T. S. Thind (ed.). CAB International, Wallingford, U.K.

Ishii, H., and Yanase, H. 2000. Venturia nashicola, the scab fungus of Japanese and Chinese pears: A species distinct from V. pirina. Mycol. Res. 104:755759 .

Jeger, M., Bragard, C., Caffier, D., Candresse, T., Chatzivassiliou, E., DehnenSchmutz, K., Gilioli, G., Gregoire, J. C., Jaques Miret, J. A., MacLeod, A., Navajas Navarro, M., Niere, B., Parnell, S., Potting, R., Rafoss, T., Urek, G., Van Bruggen, A., Van der Werf, W., West, J., Winter, S., Gonzalez-Dominguez, E., Vicent, A., Vloutoglou, I., Bottex, B., and Rossi, V. 2017. Pest categorisation of Venturia nashicola. EFSA J. 15:e05034.

Johnson, S. J. V., Jones, D., Thrimawithana, A. H., Deng, C. H., Bowen, J. K., Mesarich, C. H., Ishii, H., Bus, V. G. M., Won, K., and Plummer, K. M. 2018. Draft genome sequence of the Asian pear scab pathogen, Venturia nashicola. bioRxiv. doi: https://doi.org/10.1101/354241

Kolmogorov, M., Raney, B., Paten, B., and Pham, S. 2014. Ragout-A referenceassisted assembly tool for bacterial genomes. Bioinformatics 30:i302-309.

Li, B.-H., Yang, J.-R., Dong, X.-L., Li, B.-D., and Xu, X.-M. 2007. A dynamic model forecasting infection of pear leaves by conidia of Venturia nashicola and its evaluation in unsprayed orchards. Eur. J. Plant Pathol. 118:227-238.
MacHardy, W. E. 1996. Apple scab: Biology, epidemiology, and management. APS Press, St. Paul, Minnesota.

Min, B., Grigoriev, I. V., and Choi, I. G. 2017. FunGAP: Fungal genome annotation pipeline using evidence-based gene model evaluation. Bioinformatics 33 : 2936-2937.

Park, P., Ishii, H., Adachi, Y., Kanematsu, S., leki, H., and Umemoto, S. 2000. Infection behavior of Venturia nashicola, the cause of scab on Asian pears. Phytopathology 90:1209-1216.

Simão, F. A., Waterhouse, R. M., loannidis, P., Kriventseva, E. V., and Zdobnov, E. M. 2015. BUSCO: assessing genome assembly and annotation completeness with single-copy orthologs. Bioinformatics 31:3210-3212.

Sivanesan, A. 1974. Venturia carpophila. CMI Descriptions of Pathogenic Fungi and Bacteria 41:402.

Sivanesan, A. 1977. The taxonomy and pathology of Venturia species. Bibliotheca Mycologica, Lubrecht \& Cramer Ltd., Vaduz, Liechtenstein.

Sivanesan, A., Waller, J. M., and Mordue, J. E. M. 1974. Venturia pirina. CMI descriptions of fungi and bacteria 41:404.

Stanke, M., Keller, O., Gunduz, I., Hayes, A., Waack, S., and Morgenstern, B. 2006. AUGUSTUS: Ab initio prediction of alternative transcripts. Nucleic Acids Res. 34 : W435-W439.

Tanaka, S., and Yamamoto, S. 1964. Studies on pear scab, II. Taxonomy of the causal fungus of Japanese pear scab. Jpn. J. Phytopathol. 29:128-136.

Thomma, B. P. H. J., Seidl, M. F., Shi-Kunne, X., Cook, D. E., Bolton, M. D., van Kan, J. A. L., and Faino, L. 2016. Mind the gap; seven reasons to close fragmented genome assemblies. Fungal Genet. Biol. 90:24-30.

Walker, B. J., Abeel, T., Shea, T., Priest, M., Abouelliel, A., Sakthikumar, S., Cuomo, C. A., Zeng, Q., Wortman, J., Young, S. K., and Earl, A. M. 2014. Pilon: An integrated tool for comprehensive microbial variant detection and genome assembly improvement. PLoS One 9:e112963.

Zhang, Y., Crous, P. W., Schoch, C. L., Bahkali, A. H., Guo, L. D., and Hyde, K. D. 2011. A molecular, morphological and ecological re-appraisal of Venturiales-a new order of Dothideomycetes. Fungal Divers. 51:249-277.

Zimin, A. V., Marcais, G., Puiu, D., Roberts, M., Salzberg, S. L., and Yorke, J. A. 2013. The MaSuRCA genome assembler. Bioinformatics 29:2669-2677. 